\title{
KONGRESSKALENDER
}

\section{Mai 2020}

\subsubsection{0, Dresden}

25. Dresdner Frühjahrstagung für

Forensische Psychiatrie

Theorie und Praxis der forensischen

Psychopathologie

Dr. Lange, Prof. Dr. Bauer, Dr. Lammel, Dr. Sutarski

Information: www.forensik-dresden.de

\section{Juni 2020}

06. Juni 2020, Wien, Österreich

14. Wiener Frühjahrstagung für

Forensische Psychiatrie

Österreichische Gesellschaft für Forensische Psychiatrie

Prof. Dr. Th. Stompe, Prof. Dr. H. Schanda

Information: www.ce-management.com

09.-11.06.2020, Stockholm, Schweden

The Stockholm Criminology Symposium

Gun violence: sound knowledge and

countermeasures

Swedish National Council of Crime

Prevention

City Conference Center, Stockholm

Information: www.criminologysymposi-

um.com

22.06.2020, Berlin-Mitte

24. Berliner Junitagung für Forensische

Psychiatrie und Psychologie

Die erheblich verminderte Schuldfähig-

keit §21 StGB -

Wie lässt sie sich erfahrungswissenschaft-

lich begründen?

Forensische Psychiatrie Psychologie Berlin

e.V. (FPPB) \&

Institut für Forensische Psychiatrie der Charité

Langenbeck-Virchow-Haus

Information: www.forensik-berlin.de

(Veranstaltungen)

23.-25.06.2020, Krakau, Polen

18th Annual IAFMHS Conference

Past, Present, and Future of Forensic

Mental Health Services

Information: www.iafmhs.org

\section{Juli 2020}

16./23./30.07.2020, Gießen

Grundlagen der Sachverständigentätigkeit (Grundsätze der Sachverständigentätigkeit, Psychologisch-diagnostische Grundlagen)

Dr. Th. Wolf, A. Rohner, J. Kempfer, Dr. V. Hofstetter

Information: www.vitos.de/hainaer-forensikseminare

\section{September 2020}

01.-04.09.2020, Frankfurt am Main 16th Conference International Association for the Treatment of Sexual Offenders (IATSO)

Prof. Dr. Martin Rettenberger

Information: www.iatso.org

07.-09.09.2020, Zürich, Schweiz

12. Internationales Symposium Forensische Psychiatrie

World Trade Center Zürich

PD Dr. A. Rossegger, Prof. Dr. J. Endrass, A. Nägeli, Prof. Dr. M. Graf

Information: www.forensiktagung.ch

09.-12.09.2020, Bukarest, Rumänien

European Association of Criminology

19th Annual ESC Conference

(II)legal organizations and crime

Information: http://www.esc-eurocrim.org/

16.-19.09.2020, Cluj-Napoca, Transsylvanien, Rumänien

European Association of Psychology and

Law (EAPL)

30th Annual Conference

Information: http://eapl2020.ro/

28.-30.09.2020, Potsdam-Griebnitzsee

Begutachtungskurs Forensische

Psychiatrie:

Schwerpunkt Kriminalprognose

Dr. F. Wendt, Prof. Dr. H.-L. Kröber

Zentrum für Forensisch-Psychiatrische

Begutachtung

Information:www.zfpb.de; kroeber@zfpb.de

\section{Oktober 2020}

08.-09.10.2020, München

35. Herbsttagung für Forensische

Psychiatrie

Abteilung für Forensische Psychiatrie der

Universität München

Prof. Dr. K. Schiltz

Information: www.forensik-muenchen.de

20.-22.10.2020, Bergen, Norwegen

6th Bergen International Conference on

Forensic Psychiatry

Psychopathy

Information: www.bergenconference.no

21.-24.10.2020, San Antonio, Texas

Association for the Treatment of Sexual

Abusers (ATSA)

39th Annual Research and Treatment

Conference

Information: http://www.atsa.com/future-

atsa-conferences

21.-22.10.2020, Gießen

Die Vorhersage von Gewalttaten mit dem HCR-20 ${ }^{\mathrm{v}}$

Vitos Klinik für forensische Psychiatrie

Haina, Standort Gießen

S. Eucker

Information: www.vitos.de/hainaer-forensikseminare

22.-25.10.2020, Chicago, IL (USA)

American Academy of Psychiatry and the Law

51th Annual Meeting (with APA)

Marriott Downtown

Information: www.AAPL.org

\section{November 2020}

18.-21.11.2020 Washington D. C. (USA)

American Society of Criminology - 76th

Annual Meeting

Washington DC Marriott Marquis

Information: www.asc41.com

Hinweise für den Kongresskalender bitte an: mail@hlkroeber.de

Alle Veranstaltungen unter Vorbehalt von Absage oder Verschiebung. 